\title{
Effects of Spin-Dependent Interactions on Polarization of Bright Polariton Solitons
}

\author{
M. Sich, F. Fras, J. K. Chana, M. S. Skolnick, and D. N. Krizhanovskii \\ Department of Physics and Astronomy, University of Sheffield, Sheffield, S3 7RH, United Kingdom \\ A. V. Gorbach, R. Hartley, and D. V. Skryabin \\ Department of Physics, University of Bath, Bath, BA2 7AY, United Kingdom \\ S. S. Gavrilov \\ Institute of Solid State Physics RAS, Chernogolovka, 142432, Russia \\ E. A. Cerda-Méndez, K. Biermann, R. Hey, and P. V. Santos \\ Paul-Drude-Institut für Festkörperelektronik, 10117 Berlin, Germany
}

(Received 21 June 2013; published 28 January 2014)

\begin{abstract}
We report on the spin properties of bright polariton solitons supported by an external pump to compensate losses. We observe robust circularly polarized solitons when a circularly polarized pump is applied, a result attributed to phase synchronization between nondegenerate TE and TM polarized polariton modes at high momenta. For the case of a linearly polarized pump, either $\sigma^{+}$or $\sigma^{-}$circularly polarized bright solitons can be switched on in a controlled way by a $\sigma^{+}$or $\sigma^{-}$writing beam, respectively. This feature arises directly from the widely differing interaction strengths between co- and cross-circularly polarized polaritons. In the case of orthogonally linearly polarized pump and writing beams, the soliton emission on average is found to be unpolarized, suggesting strong spatial evolution of the soliton polarization. The observed results are in agreement with theory, which predicts stable circularly polarized solitons and unstable linearly polarized solitons.
\end{abstract}

DOI: 10.1103/PhysRevLett.112.046403

Introduction.-Solitons are broadly investigated across many areas of physics, including nonlinear optics [1] and cold atom physics $[2,3]$. Optical and matter wave solitons form when photon or particle interactions are able to compensate for the dispersive spreading of wave packets. The spin or polarization degree of freedom gives rise to the richness of nonlinear effects. Temporal vector solitons consisting of two polarization components have been investigated in birefringent fiber cavity lasers [4] and nonlinear lattices [5]. The realization of the spinor atom BEC stimulated the theoretical investigation of a variety of phenomena such as spin textures and vortices [6,7], magnetic solitons [8,9], and bright-dark soliton complexes [10], which arise from spin-dependent atomic scattering.

Strong exciton-photon coupling in semiconductor microcavities enables the formation of half-light half-matter bosonic quasiparticles (2D polaritons) [11,12]. Polaritons have been used to demonstrate condensation [13], superfluidity [14], and dark [15] and bright [16-19] solitons. Nonlinear spin-dependent interactions of co- and crosscircularly polarized photons may have different strengths [20]. Conversely, interactions of co- and cross-circularly polarized polaritons not only differ in strength but also have opposite signs such that polaritons with parallel spins repel, while those with opposite spins attract [11]. The interplay of the two polariton spin components naturally leads to a variety
PACS numbers: 71.36.+c, 42.65.Pc, 42.65.Tg, 78.55.Cr

of possible nonlinear states, expanding the possibilities to control the dynamics and response to external stimuli. Spin multistability [21] of exciton-polariton states has recently been reported [22,23], expanding on previous research into nonlinear polarization effects in optical resonators $[21,24]$. Polariton spin switching [25-27], and more recently, conservative (i.e., without gain) dark polariton half-solitons (vector solitons) were also observed [28].

The bright polariton solitons observed recently [18] can be switched on and off on a picosecond time scale and have well-defined size $(2-4 \mu \mathrm{m})$ and amplitude. Importantly, polariton-polariton interactions are $2-3$ orders of magnitude larger than the nonpolaritonic ones achieved in the weak coupling regime. In this Letter, we investigate the spin properties of polariton solitons, which may create opportunities in ultrafast all-optical digital signal processing $[29,30]$, where spin-based architectures have considerable potential [31,32]. Furthermore, we report the symmetry breaking in the vector polariton soliton system arising from spin-dependent interparticle interactions.

The polariton solitons propagate in a dissipative environment where losses are fully compensated by gain from an external continuous wave (cw) pump permitting the scalability of possible polariton circuits [32,33]. We find that when the background cw pump and the local pulsed writing beam (WB) which triggers the soliton [see Fig. 1(a)] 

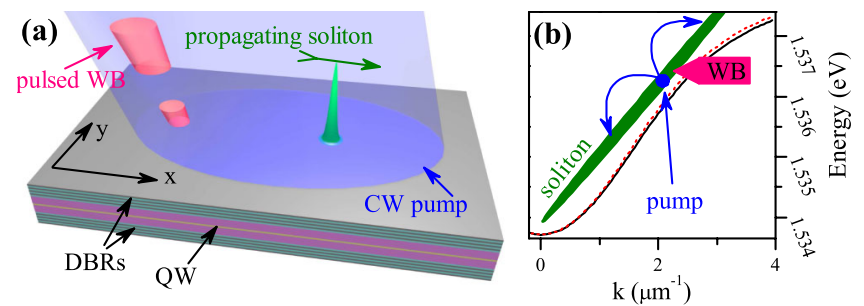

FIG. 1 (color online). (a) Schematic of soliton excitation in a microcavity consisting of distributed Bragg reflectors (DBRs) and a quantum well (QW). The cw pump and pulsed WB are incident along the $X$ direction. (b) Schematic of soliton spectrum and excitation in $E-k$ space with TE (dotted) and TM (solid) polarized polariton dispersions.

are circularly copolarized, solitons with the same polarization are readily excited. Once triggered, the soliton maintains its polarization during propagation over macroscopic distances, indicating phase synchronization between the nondegenerate TE and TM polarized polariton modes. If the pump is linearly polarized, then solitons with either $\sigma^{+}$or $\sigma^{-}$ circular polarization can be triggered by a WB with the corresponding polarization due to the very different interaction strengths between co- and cross-circularly polarized polaritons. Theoretically, we find that linearly polarized solitons are unstable. As a result, when both the pump and WB are linearly polarized, either the soliton polarization evolves dynamically or only a circularly polarized soliton is excited. Details of the experimental arrangements required for soliton observation in the GaAs-based microcavity sample can be found in the Supplemental Material [34] and Ref. [18].

Numerical model.-To support our experimental observations, we use the well-known equations for the amplitudes of excitonic oscillators with positive and negative spins [18]:

$\partial_{t} \psi_{ \pm}+\left(\gamma_{e}-i \delta_{e}+i\left|\psi_{ \pm}\right|^{2}-i V\left|\psi_{\mp}\right|^{2}\right) \psi_{ \pm}=i \Omega_{R} E_{ \pm}$.

Here, $V=0.05$ is the strength of the attraction between oscillators with opposing spins relative to the repulsion between those with like spins, $\gamma_{e}$ is the coherence decay rate, and $\delta_{e}$ is the detuning of the excitonic resonance from the frequency of the pump field. $\Omega_{R}$ is the Rabi frequency, which couples the excitonic equations to the amplitudes $E_{ \pm}$ of the $\sigma^{ \pm}$polarized components of the electric field. True photonic modes of the cavity are the TE (subscript $y$ ) and TM (subscript $x$ ) modes. The amplitudes of these modes obey the following set of equations:

$$
\begin{aligned}
& \partial_{t} E_{x, y}-i \nabla^{2} E_{x, y}+\left(\gamma_{p}-i \delta_{p}\right) E_{x, y} \\
& =i \Omega_{R} \psi_{x, y}+(a \pm b) E_{p} e^{i k_{p} x} .
\end{aligned}
$$

$E_{x, y}$ are linked to the amplitudes of the circularly polarized components as $E_{x, y} \simeq\left(l_{ \pm} / \sqrt{2}\right)\left(E_{+} \pm E_{-}\right)$, $\psi_{x, y} \simeq\left(l_{ \pm} / \sqrt{2}\right)\left(\psi_{+} \pm \psi_{-}\right), l_{+}=1, l_{-}=i$, see Ref. [35] for details. In Eq. (2), $E_{p}$ and $k_{p}$ are the pump amplitude and transverse momentum, while $\gamma_{p}$ and $\delta_{p}$ are the photon decay rate and detuning. Relative values of $a$ and $b$ $\left(|a|^{2}+|b|^{2}=1\right)$ control the pump polarization.

Pump and WB circularly polarized: Experiment and theory. - If the polariton resonance is driven by a slightly blueshifted $\mathrm{cw}$ laser, the internal pump polariton field exhibits bistable behavior as a function of the pump power [36,37]. Polariton solitons [16,18] can be considered as spatially localized excitations from a low intensity to a high intensity cw pump state of the bistability loop. Furthermore, the polariton-polariton stimulated parametric scattering process (modulation instability) from the switched-on pump state also ensures that soliton harmonics with a broad range of $k$ vectors are populated [Fig. 1(b)]. Using our previous theoretical predictions [16], we tune the system into the bistability domain of its pump state and apply a cocircularly polarized WB, which excites a soliton. Experimentally measured spatiotemporal trajectories of such solitons and the temporal evolution of their circular polarization degree (CPD) $\rho_{c}$ defined as $\rho_{c}=\left(\left|E_{+}\right|^{2}-\right.$ $\left.\left|E_{-}\right|^{2}\right) /\left(\left|E_{+}\right|^{2}+\left|E_{-}\right|^{2}\right)$ are shown in Figs. 2(a)-2(c). A high CPD of the soliton [Fig. 2(c)] is obtained with negligible emission in the opposing circular polarization component. Thus, when the pump and WB are circularly copolarized, polariton-polariton scattering to the soliton harmonics occurs only in that polarization.

The full width half maximum of the soliton is measured to be $\sim 6-7 \mu \mathrm{m}$, which is close to the resolution of our setup

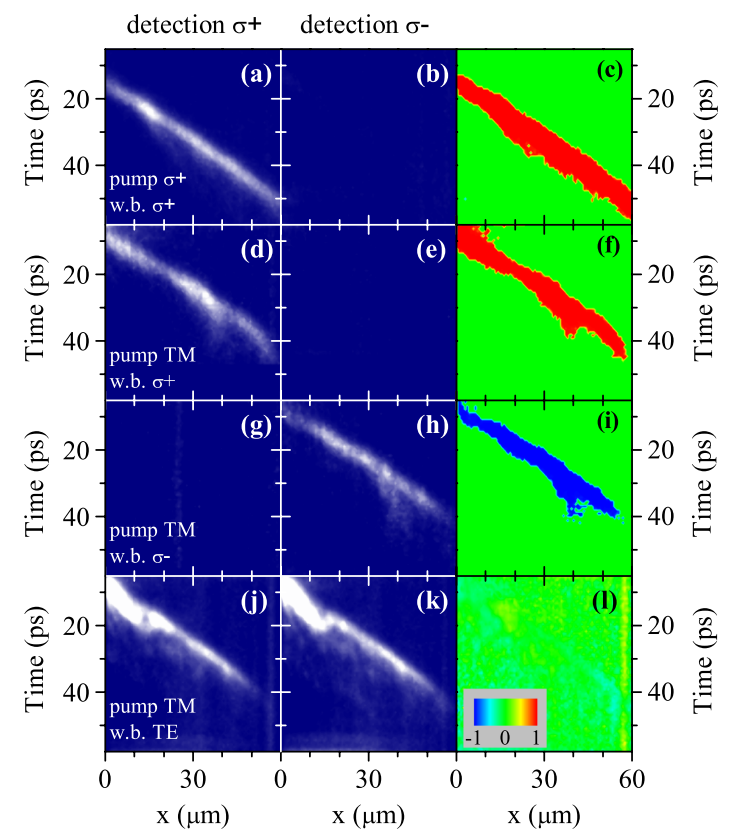

FIG. 2 (color online). Soliton emission intensity recorded as a function of time and position across the sample in $\sigma^{+}$(a),(d), (g), (j) and $\sigma^{-}$(b),(e),(h),(k) circular polarizations. Soliton CPD $\rho_{c}$ as a function of time and position (c),(f),(i),(l). The pump polarization is $\sigma^{+}$in (a)-(c) and TM linear in (d)-(1). The WB polarization is $\sigma^{+}$in (a)-(f), $\sigma^{-}$in (g)-(i), and TE linear in (j)-(1). The WB arrives at time $t=0 \mathrm{ps}$ at position $x \sim-10 \mu \mathrm{m}$. 
$(5 \mu \mathrm{m})$. We note that at low excitation densities, there is a TE-TM splitting of polariton modes of $\sim 0.1 \mathrm{meV}$ at high $k$ vectors. This splitting is responsible, for example, for the optical spin Hall effect [38] and for the formation of dark spinor polariton solitons [28]. In a nonsolitonic linear regime, the polarization of a polariton wave packet excited at finite $k$ vectors would oscillate between $\sigma^{+}$and $\sigma^{-}$with a period of $\sim 20 \mathrm{ps}$. The fact that the $\rho_{c}$ is $\sim 0.9$ over the whole duration of the soliton pulse $\sim 35$ ps [Fig. 2(c)] indicates effective quenching of the TE-TM splitting. Phase synchronization [39] between the interacting TE and TM modes occurs, since the soliton potential energy $(\sim 0.3-0.5 \mathrm{meV})$ is greater than the TE-TM splitting [18]. Therefore, to some extent, $\sigma^{ \pm}$polarized polariton solitons resemble vector solitons in birefringent optical fibers [4] when the group and phase velocities of cross-linearly polarized modes lock together, although the mechanism of polariton soliton formation is very different.

In numerical modeling, a circularly polarized pump is defined by setting $b=0$. If we take into account the TETM splitting of $\sim 0.1 \mathrm{meV}$ at $k$ vectors $k \sim k_{p}$, the numerical simulations show excitation of circularly polarized solitons maintaining their polarization during propagation, in agreement with the experiment. Therefore, for simplicity, in the theory analysis which follows next, we disregarded this splitting [40].

Pump linearly polarized: Theory analysis of soliton stability.-Now we change the pump polarization to linear, which corresponds to $b=a$ in Eq. (2), and tune our system into the bistability domain of the linearly polarized state. It was predicted and observed [21,23,41-43] that if polaritons at nearly zero momentum are driven resonantly by a linearly polarized beam, then different polarization states ( $\sigma^{ \pm}$and linear) of the spatially extended (nonsolitonic) intracavity field can all be stable in a finite range of pump powers. The situation is qualitatively different for polariton solitons. The thin lines in Fig. 3(a) show how the CPD of the internal homogeneous polariton field extending over the whole excitation pump spot (nonsolitonic regime) depends on $E_{p}$. Linearly $\left(\rho_{c}=0\right)$, elliptically, and quasicircularly polarized homogeneous polariton states may be excited for the same amplitude of the linearly polarized pump. The $\rho_{c}$, $E_{p}$ relationship is symmetric with respect to $\rho_{c} \rightarrow-\rho_{c}$ due to the symmetry $\left(E_{+}, E_{-}\right) \rightarrow\left(E_{-}, E_{+}\right)$. The quasicircularly polarized spatially homogeneous states with $\rho_{c}$ close to \pm 1 are the only ones which are stable relative to perturbations with momenta equal to the pump momentum. To find spatially localized soliton solutions, we transform Eqs. (1) and (2) into a reference frame moving with an unknown velocity $v$, and by taking $\partial_{t}=\partial_{y}=0$, we reduce them to a set of differential equations with respect to the new coordinate $\xi=x-v t$. We then solve the resulting equations numerically and find the soliton profiles and associated velocities self-consistently [18]. The branches of the stable (quasicircularly polarized) and unstable (linearly and elliptically polarized) solitons are plotted in Fig. 3(a) as
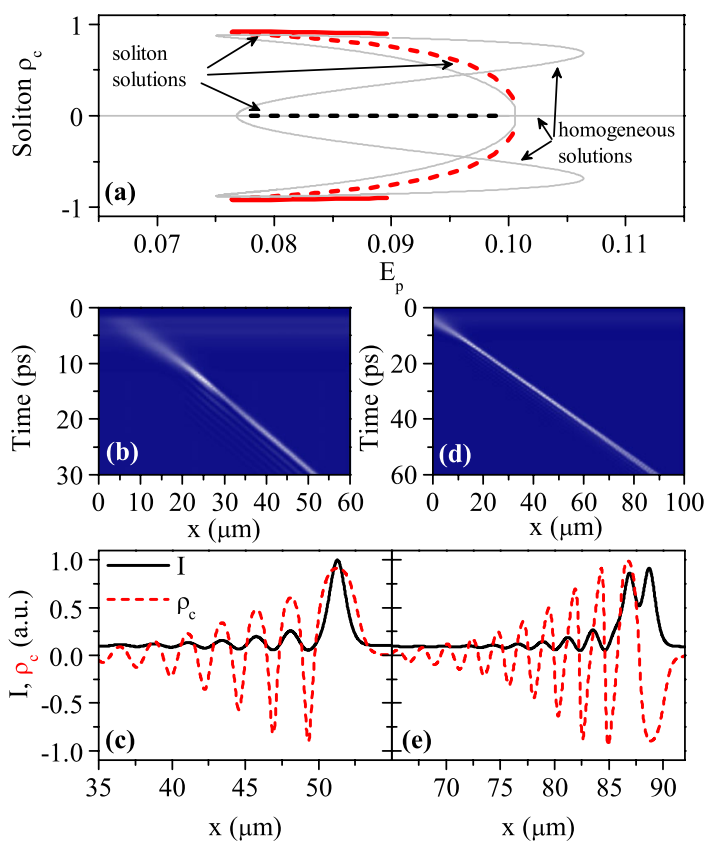

FIG. 3 (color online). (a) Thin grey lines show the CPD $\rho_{c}$ of polariton field vs the pump amplitude corresponding to a homogeneous pump polariton field. The dashed (solid) lines correspond to unstable (stable) soliton branches. (b),(d) Spatiotemporal trajectories of solitons when the pump and WB are orthogonally (b) and parallelly (d) linearly polarized. (c),(e) Soliton intensities and CPD $\rho_{c}$ as a function of position at time $t=30 \mathrm{ps}$ (c) and $60 \mathrm{ps}$ (e) after the WB pulse.

thick full and dashed lines, respectively. While repulsive interactions between cocircularly polarized polaritons keep the soliton population in resonance with the pump, the attractive coupling between $\sigma^{+}$and $\sigma^{-}$polarized polaritons pulls the system out of resonance. This makes linearly polarized solitons unstable, resulting in a breakup into circularly polarized components. Our numerical analysis shows that if the cross-spin interaction is repulsive, then linearly polarized solitons become stable.

Pump linearly polarized, WB circularly polarized: Experiment.-Stable solitons with $\sigma^{+}\left(\sigma^{-}\right)$circular polarization have been experimentally observed when a TM linearly polarized pump and a $\sigma^{+}\left(\sigma^{-}\right)$circularly polarized WB are applied. Figures 2(d), 2(e), 2(g), and 2(h) show spatiotemporal traces of the soliton intensities measured in $\sigma^{+}$[Figs. 2(d) and 2(g)] and $\sigma^{-}$[Figs. 2(e) and 2(h)] polarizations. We clearly observe either $\sigma^{+}$or $\sigma^{-}$polarized soliton emission depending on the state of the WB. The measured $\rho_{c}$ for solitons is close to \pm 0.9 in both cases, as shown in Figs. 2(f) and 2(i). When the $\sigma^{+}\left(\sigma^{-}\right)$polarized WB arrives, it causes a blueshift of only the $\sigma^{+}\left(\sigma^{-}\right)$ polariton energies, bringing them into resonance with the pump. As a result, pure $\sigma^{+}\left(\sigma^{-}\right)$polarized solitons are turned on. Importantly, this effect is due to spin anisotropy in polariton-polariton interactions. This agrees with our simulations, which show that if the spin-dependent interactions were fully isotropic $(V=1)$, then solitons 
copolarized with the pump would be triggered independently of the WB polarization.

Pump and WB linearly polarized: Theory and experiment.-When the polarizations of the pump and WB are orthogonal, the simulations show that instability of the linearly polarized solitons results in the development of either $\sigma^{+}$or $\sigma^{-}$quasicircularly polarized single solitons. These states can be excited with equal probabilities as the phases of the WB and pump are uncorrelated. An example of the calculated trajectory of a quasicircularly $\sigma^{+}$ polarized soliton is shown in Fig. 3(b). Figure 3(c) shows the spatial distribution of the soliton intensity and CPD $\rho_{c}$ at time 30 ps. $\rho_{c}$ is close to 1 at the maximum of the soliton intensity, so that on average, the soliton is circularly polarized. By contrast, in the case of a quasiparallel pump and WB polarizations, numerical modeling revealed the development of a double-hump soliton, where the polarization changes from $\sigma^{+}$to $\sigma^{-}$between the intensity maxima on length scale of $2 \mu \mathrm{m}$ [see Figs. 3(d) and 3(e)].

Experimentally, we were able to observe robust solitonic propagation only when the polarizations of the pump and WB were orthogonal [44]. The trajectories of such solitons detected in the $\sigma^{+}$and $\sigma^{-}$polarizations are shown in Figs. 2(j) and 2(k), respectively. The corresponding $\rho_{c}$ remains near zero as a function of time and position as shown in Fig. 2(1). Similar results are observed for the measurements in the bases of horizontal $(X)$, vertical $(Y)$, and diagonal $(D 1-D 2)$ linear polarizations. The total polarization degree $\rho=\sqrt{\rho_{c}^{2}+\rho_{l}^{2}}\left(\rho_{l}\right.$ is the linear polarization degree) averaged over $10^{8}$ pulses fluctuates near zero over the duration of the pulse. The correlation measurements between different polarization components of the soliton emission (see Supplemental Material [34]) suggest that $\rho_{c}(x)$ as well as $\rho_{l}(x)$ undergo rapid oscillations across the soliton profile similar to those in Fig. 3(e) so that on average, $\rho_{c, l}^{\text {int }} \sim 0$. Such oscillations may also have a random phase from pulse to pulse, leading to no observable spatial dependence of $\rho_{c, l}$ across the soliton profile averaged over many soliton pulses as in Fig. 2. We note that high-order polarization locked vector solitons with single- and doublehump profiles in crossed linear polarizations were reported in fiber lasers [45]. However, to the best of our knowledge, such symmetry breaking when there is a spatial separation of trajectories of $\sigma^{+}$and $\sigma^{-}$polariton solitons due to attractive $\sigma^{+}-\sigma^{-}$coupling [Fig. 3(e)] was not observed in optics or cold atoms.

Dependence of soliton CPD on pump polarization: Experiment and theory.-We have also performed a systematic study of the soliton CPD $\rho_{c}$ as a function of the pump CPD $\rho_{p}$ for the cases of $\sigma^{+}, \sigma^{-}$, and linearly polarized WBs. The pump power was fixed at a maximum value of $\sim 120 \mathrm{~mW}$. The results summarized in Fig. 4(a) show that $\sigma^{+}\left(\sigma^{-}\right)$polarized spatially localized pulses can be excited in the range of $\rho_{p}$ from 1 to -0.25 ( -1 to 0.25 )

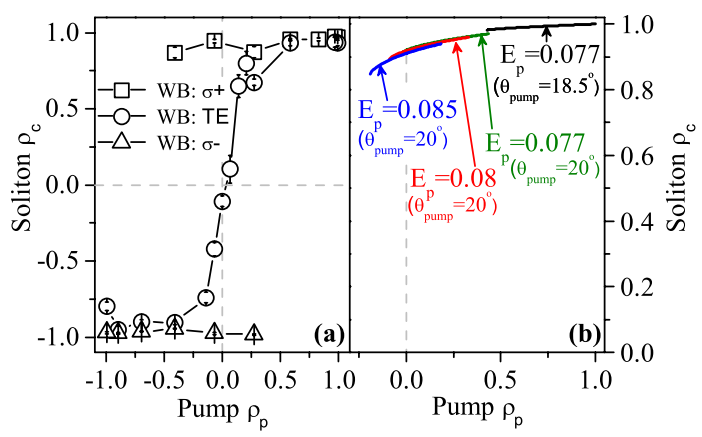

FIG. 4 (color online). (a) Soliton $\mathrm{CPD} \rho_{c}$ as a function of pump CPD $\rho_{p}$ recorded for the case of $\sigma^{+}, \sigma^{-}$circularly polarized, and TE linearly polarized WB (circles, squares, and triangles, respectively). (b) Numerical modeling of soliton CPD $\rho_{c}$ as a function of $\rho_{p}$ recorded for the case of $\sigma^{+}$polarized WB for different pump amplitudes and different pump angles.

using $\sigma^{+}\left(\sigma^{-}\right)$polarized WBs. Thus, solitons can be excited under a linearly or circularly polarized pump at the same pump power. There is a range $\rho_{p}$ from about -0.25 to 0.25 when both $\sigma^{+}$and $\sigma^{-}$polarized solitons can be triggered. For the linearly polarized $\mathrm{WB}$, the slight bias of $\rho_{p}$ towards $\sigma^{+}$or $\sigma^{-}$polarizations by 0.1 quickly ensures excitation of either $\sigma^{+}$or $\sigma^{-}$circularly polarized solitons with $|\rho| \sim 0.85-0.95$, implying the stability of circularly polarized solitons if the symmetry is slightly disturbed. Numerically, we only found exact stable solitons in a narrower interval of $\rho_{p}$ at a fixed pump power and pump angle [see Fig. 4(b)]. In theory, in order to observe soliton emission at lower $\rho_{p}$, it is necessary to increase the pump field $E_{p}$ or the pump angle $\Theta_{\text {pump }}$. (Increasing $\Theta_{\text {pump }}$ brings the cw pump closer to resonance and, hence, reduces the bistability threshold.) This is understandable since the bistability region in which the soliton forms is expected to shift to higher pump powers as $\rho_{p}$ decreases due to spin-dependent anisotropy in polariton-polariton interactions. Taking into account the coupling of coherent excitons to the incoherent exciton reservoir through the formation and decay of biexcitons $[22,41,46,47]$ may lead to a better agreement between theory and experiment [34].

In conclusion, the phase synchronization between TE and TM polarized polaritons ensures the propagation of circularly polarized solitons. Stable $\sigma^{+}$or $\sigma^{-}$polarized solitons are observed under linearly polarized pumping, as the instability of linearly polarized solitons leads to the fast evolution of the soliton polarization in space-time. Finally, vector solitons can potentially be studied in external magnetic fields, which may allow the tuning of soliton group velocities in $\sigma^{+}$and $\sigma^{-}$polarizations and control of the relative soliton trajectories.

We acknowledge support from the EPSRC Grant No. EP/J007544/1 and EU ITN grant "Clermont 4." D. V.S. and A. V.G. acknowledge support from the Leverhulme Trust. S.S. G. acknowledges support from grant RFBR 12-02-00521. 
[1] T. Ackemann et al., Advances in Atomic, Molecular and Optical Physics, edited by E. Arimondo P. R. Berman, and C. C. Lin (Academic Press, New York, 2009), Vol. 57, pp. 323-421.

[2] L. Khaykovich, F. Schreck, G. Ferrari, T. Bourdel, J. Cubizolles, L. D. Carr, Y. Castin, and C. Salomon, Science 296, 1290 (2002).

[3] K. E. Strecker, G. B. Partridge, A. G. Truscott, and R. G. Hulet, Nature (London) 417, 150 (2002).

[4] S. T. Cundiff, B. C. Collings, N. N. Akhmediev, J. M. Soto-Crespo, K. Bergman, and W. H. Knox, Phys. Rev. Lett. 82, 3988 (1999).

[5] Y. V. Kartashov, B. A. Malomed, V. A. Vysloukh, and L. Torner, Opt. Lett. 34, 3625 (2009).

[6] K. Kasamatsu, M. Tsubota, and M. Ueda, Phys. Rev. A 71, 043611 (2005).

[7] Y. Kawaguchi, H. Saito, and M. Ueda, Phys. Rev. Lett. 96, 080405 (2006).

[8] Z. D. Li, P. B. He, L. Li, J. Q. Liang, and W. M. Liu, Phys. Rev. A 71, 053611 (2005).

[9] M. Wang, B. Tian, W.-R. Shan, X. Lü, and Y.-S. Xue, Nonlinear Dynamics 69, 1137 (2012).

[10] H. E. Nistazakis, D. J. Frantzeskakis, P. G. Kevrekidis, B. A. Malomed, and R. Carretero-Gonzalez, Phys. Rev. A 77, 033612 (2008).

[11] A. Kavokin et al., Microcavities (Oxford University Press, New York, 2007).

[12] D. Sanvitto and V.B. Timofeev, Exciton Polaritons in Microcavities: New Frontiers, Solid State Sciences, Vol. 172 (Springer, New York, 2012).

[13] J. Kasprzak et al., Nature (London) 443, 409 (2006).

[14] A. Amo, J. Lefrère, S. Pigeon, C. Adrados, C. Ciuti, I. Carusotto, R. Houdré, E. Giacobino, and A. Bramati, Nat. Phys. 5, 805 (2009).

[15] A. Amo et al., Science 332, 1167 (2011).

[16] O. A. Egorov, D. V. Skryabin, A. V. Yulin, and F. Lederer, Phys. Rev. Lett. 102, 153904 (2009).

[17] A. V. Gorbach, B. A. Malomed, and D. V. Skryabin, Phys. Lett. A 373, 3024 (2009).

[18] M. Sich, D. N. Krizhanovskii, M. S. Skolnick, A. V. Gorbach, R. Hartley, D. V.Skryabin, E. A. Cerda-Méndez, K. Biermann, R. Hey, and P. V. Santos, Nat. Photonics 6, 50 (2012).

[19] D. Tanese et al., Nat. Commun. 4, 1749 (2013).

[20] R. W. Boyd, Nonlinear Optics (Academic Press, New York, 2003).

[21] N. A. Gippius, I. A. Shelykh, D. D. Solnyshkov, S. S. Gavrilov, Y. G. Rubo, A. V. Kavokin, S. G. Tikhodeev, and G. Malpuech, Phys. Rev. Lett. 98, 236401 (2007).

[22] D. Sarkar, S. S. Gavrilov, M. Sich, J. H. Quilter, R. A. Bradley, N. A. Gippius, K. Guda, V. D. Kulakovskii, M. S. Skolnick, and D. N. Krizhanovskii, Phys. Rev. Lett. 105, 216402 (2010).

[23] T. K. Paraïso, M. Wouters, Y. Léger, F. Morier-Genoud, and B. Deveaud-Plédran, Nat. Mater. 9, 655 (2010).

[24] M. Kitano, T. Yabuzaki, and T. Ogawa, Phys. Rev. Lett. 46, 926 (1981).

[25] A. Amo, T. C. H. Liew, C. Adrados, R. Houdré, E. Giacobino, A. V. Kavokin, and A. Bramati, Nat. Photonics 4, 361 (2010).

[26] C. Adrados, T. C. H. Liew, A. Amo, M. D. Martín, D. Sanvitto, C. Antón, E. Giacobino, A. Kavokin, A. Bramati, and L. Viña, Phys. Rev. Lett. 107, 146402 (2011).

[27] S. S. Gavrilov, A. V. Sekretenko, N. A. Gippius, C. Schneider, S. Höfling, M. Kamp, A. Forchel, and V. D. Kulakovskii, Phys. Rev. B 87, 201303 (2013).
[28] R. Hivet et al., Nat. Phys. 8, 724 (2012).

[29] S. Barland et al., Nature (London) 419, 699 (2002).

[30] F. Pedaci et al., Appl. Phys. Lett. 92, 011101 (2008).

[31] I. Zutic, J. Fabian, and S. Das Sharma, Rev. Mod. Phys. 76, 323 (2004).

[32] T. C. H. Liew, A. V. Kavokin, and I. A. Shelykh, Phys. Rev. Lett. 101, 016402 (2008).

[33] Polariton bright solitons have a considerable advantage over polariton spin bullets, which are decaying and diffracting nonsolitonic wave packets [26].

[34] See Supplemental Material at http://link.aps.org/ supplemental/10.1103/PhysRevLett.112.046403 for details of the experimental setup and the correlation measurements of solitons produced with a linearly polarized pump and WB.

[35] While TE photonic and excitonic modes can be represented exactly as $E_{y}=(i / \sqrt{2})\left(E_{+}-E_{-}\right), \psi_{y}=(i / \sqrt{2})\left(\psi_{+}-\psi_{-}\right)$, the similar transformation for TM modes is only approximately valid, since the TM mode has a nonzero component perpendicular to the cavity plane, which does not couple to excitons. However, the external incident pump angle of $\sim 20^{\circ}$, which is used here, corresponds to a small internal angle of $\sim 6^{\circ}$ due to the high refractive index of GaAs. Therefore, the nonzero component of the electric field perpendicular to the cavity plane is negligible, so the circularly polarized pump excites intracavity modes, which can be considered as circularly polarized.

[36] A. Tredicucci, Y. Chen, V. Pellegrini, M. Borger, and F. Bassani, Phys. Rev. A 54, 3493 (1996).

[37] A. Baas, J. P. Karr, H. Eleuch, and E. Giacobino, Phys. Rev. A 69, 023809 (2004).

[38] C. Leyder, M. Romanelli, J. Ph. Karr, E. Giacobino, T. C. H. Liew, M. M. Glazov, A. V. Kavokin, G. Malpuech, and A. Bramati, Nat. Phys. 3, 628 (2007).

[39] P. Walker, T. C. H. Liew, D. Sarkar, M. Durska, A. P. D. Love, M. S. Skolnick, J. S. Roberts, I. A. Shelykh, A. V. Kavokin, andD. N. Krizhanovskii, Phys. Rev. Lett. 106, 257401 (2011).

[40] If TE-TM splitting between polariton modes is included in our simulations, we find that the TE-TM splitting in the soliton regime is also quenched when the pump and WB are linearly polarized.

[41] M. Wouters, T. K. Paraïso, Y. Léger, R. Cerna, F. MorierGenoud, M. T. Portella-Oberli, and B. Deveaud-Plédran, Phys. Rev. B 87, 045303 (2013).

[42] S. S. Gavrilov, N. A. Gippius, S. G. Tikhodeev, and V. D. Kulakovskii, J. Exp. Theor. Phys. 110, 825 (2010).

[43] S. S. Gavrilov, A. V. Sekretenko, S. I. Novikov, C. Schneider, S. Höfling, M. Kamp, A. Forchel, and V. D. Kulakovskii, Appl. Phys. Lett. 102, 011104 (2013).

[44] We were not able to observe solitons when the polarizations of the pump and WB were parallel, probably because of the increased WB and pump absorption due to the excitation of the biexcitons, which can only be excited with two colinearly polarized photons.

[45] D. Y. Tang, H. Zhang, L. M. Zhao, and X. Wu, Phys. Rev. Lett. 101, 153904 (2008).

[46] S. S. Gavrilov, A. S. Brichkin, A. A. Dorodnyi, S. G. Tikhodeev, N. A. Gippius, and V.D. Kulakovskii, J. Exp. Theor. Phys. Lett. 92, 171 (2010).

[47] S.S. Gavrilov, A.S. Brichkin, A. A. Demenev, A. A. Dorodnyy, S. I. Novikov, V. D. Kulakovskii, S. G. Tikhodeev, and N. A. Gippius, Phys. Rev. B 85, 075319 (2012). 